\title{
X-ray sources in Galactic globular clusters and old open clusters
}

\author{
Maureen van den Berg \\ Harvard-Smithsonian Center for Astrophysics, 60 Garden Street, Cambridge, MA 02138, USA \\ email: mvandenberg@cfa.harvard.edu
}

\begin{abstract}
The features and make up of the population of X-ray sources in Galactic star clusters reflect the properties of the underlying stellar environment. Cluster age, mass, stellar encounter rate, binary frequency, metallicity, and maybe other properties as well, determine to what extent we can expect a contribution to the cluster X-ray emission from low-mass X-ray binaries, millisecond pulsars, cataclysmic variables, and magnetically active binaries. Sensitive X-ray observations with XMM-Newton and certainly Chandra have yielded new insights into the nature of individual sources and the effects of dynamical encounters. They have also provided a new perspective on the collective X-ray properties of clusters, in which the X-ray emissivities of globular clusters and old open clusters can be compared to each other and to those of other environments. I will review our current understanding of cluster X-ray sources, focusing on star clusters older than about 1 Gyr, illustrated with recent results.
\end{abstract}

Keywords. binaries: close, X-rays: binaries, globular clusters, open clusters and associations

\section{Introduction}

X-ray observations of old star clusters efficiently select the populations of close interacting binaries. X-ray studies can serve as a tool to study cluster dynamics: the high stellar densities can give rise to stellar encounters that may destroy or modify existing binaries, or create new ones that are rare in the field of the Galaxy. With X-ray studies we can also investigate specific phases of binary evolution; the known distance and age are helpful for piecing together a binary's past and predict its future. I review several recent results on X-ray sources in old ( $\gtrsim 1 \mathrm{Gyr}$ ) Galactic star clusters - both globular clusters and old open clusters - and briefly summarize earlier work to provide context. I cover the following subjects: observational studies of luminous X-ray sources in globular clusters ( $\gtrsim 10^{36} \mathrm{erg} \mathrm{s}^{-1}$; Section 2) and of sources fainter than $\sim 10^{33} \mathrm{erg} \mathrm{s}^{-1}$ (Section 3), and a comparison of the X-ray emissivity of old stellar populations (Section 4). Verbunt \& Lewin (2006) give a comprehensive overview of globular-cluster X-ray sources, and more recent updates can be found in, e.g., Heinke (2010), Pooley (2016), and Ivanova et al. (2017). Van den Berg (2013) gives an overview of X-ray sources in old open clusters.

\section{Luminous $X$-ray sources and transients}

The first X-ray sources in globular clusters were discovered in the early seventies, when the UHURU and OSO-7 satellites made the first systematic scans of the X-ray sky (e.g. Giacconi et al. 1974, Clark et al. 1975). Based on their X-ray luminosities of $\sim 10^{36} \mathrm{erg} \mathrm{s}^{-1}$ or higher, and their X-ray variability, these cluster sources were thought to be compact objects - neutron stars or black holes - accreting from a companion in low-mass X-ray binaries (LMXBs). Such systems had already been found in other parts of the Galaxy (see Blumenthal \& Tucker (1974) for an early review). The brief bursts of 
X-rays (Grindlay \& Heise 1975, Clark et al. 1976), sometimes detected on top of more steady X-ray emission, revealed that the compact objects had to be neutron stars (see e.g. review by Lewin et al. 1995). By considering the total mass enclosed in the globular clusters of our Galaxy, it was soon recognized that the number of bright X-ray sources associated with globular clusters is disproportionately high (Katz 1975). Since then, the luminous X-ray sources in globular clusters have been linked to dynamical interactions (tidal capture, collisions, exchange encounters; see Verbunt \& Lewin 2006 for references) that must be operating uniquely in dense environments. Verbunt \& Hut (1987) calculated the encounter number $\Gamma$, or the volume integral (over the cluster core) of $\rho_{c}^{2} / v$ where $\rho_{c}$ is the central density and $v$ is the velocity dispersion. The observations clearly support a correlation between $\Gamma$ and whether a cluster hosts a bright X-ray source or not, both under the simplifying assumptions for the cluster structural parameters made initially, and with a more sophisticated approach (Bahramian et al. 2013). $\Gamma$ has also been found to scale with the number of radio millisecond pulsars (MSPs; Bahramian et al. 2013), which are the descendants of LMXBs, in contrast to the findings by Bagchi et al. (2011) who used a more simple calculation for $\Gamma$.

At the time of writing, we know of 21 luminous $\left(L_{X} \gtrsim 10^{36} \mathrm{erg} \mathrm{s}^{-1}\right) \mathrm{X}$-ray sources in fifteen globular clusters. These include eight persistently bright sources (in seven clusters), and thirteen transients (in eight clusters) that have been observed to switch between a faint quiescent and a bright state (or vice versa) at least once. Table 1 gives an overview with various updates compared to the compilation by Bahramian et al. (2014). Objects in the growing class of very faint X-ray transients (VFXTs; not included in the table) reach peak luminosities of $\sim 10^{34-36} \mathrm{erg} \mathrm{s}^{-1}$ (Wijnands et al. 2006). It is currently not clear whether the VFXTs, identified in the Galactic Center region but also in globular clusters (Heinke et al. 2009, Arnason et al. 2015) form a homogeneous class, and why their outbursts only reach the observed levels.

For a long time it was assumed that a single X-ray source is responsible for the bright $\mathrm{X}$-ray emission from a globular cluster. The unprecedented spatial resolution of the Chandra X-ray Observatory has shown this assumption to be false. Using Chandra, White \& Angelini (2001) resolved the bright source in M 15 into two bright sources, thereby solving a long-standing puzzle regarding the source's seemingly conflicting characteristics. Rapid-response Chandra observations to follow up on triggers from X-ray satellites with lower spatial resolution have also revealed that globular clusters can host multiple transient LMXBs, such as in Terzan 5 and NGC 6440 (see references in Pooley 2016). In some cases, the quiescent counterpart of a bright transient had already been identified in deep pre-outburst Chandra images - but, due to limited X-ray sensitivity, not always. The latest transient is located in Liller 1, one of the most dense and most massive globular clusters in the Galaxy. Liller 1 was already known to host at least one bright transient, viz. the Rapid Burster. In 2018, an X-ray transient went off in Liller 1 that did not show the characteristic behavior of the Rapid Burster. With Chandra, it was found that the position of the new transient coincided with none of the known sources (Homan et al. 2018). This is an excellent example of the power of Chandra: the two X-ray binaries are separated by only $1.4^{\prime \prime}$. The recent transient and X-ray millisecond pulsar IGR J18245-2452 in M 28 has been ground breaking in the sense that its crossidentificaton with the known radio millisecond pulsar M 28 I provided the first observational proof for the long-assumed formation scenario for millisecond pulsars, in which an old neutron star is spun up by accretion in an LMXB (Papitto et al. 2013). Another interesting system is the transient IGR J17361-4441 in NGC 6388, with an exceptionally hard X-ray spectrum for a neutron-star LMXB. Del Santo et al. (2014) suggested that the transient 
originated from the tidal disruption of a planet by a white dwarf, but an LMXB nature cannot be excluded (Wijnands et al. 2015).

Small-number statistics and, in some cases, poorly known observational selection biases (e.g. related to ill-constrained outburst duty cycles, as discussed in e.g. Carbone \& Wijnands 2019), complicate the comparison of the population of bright LMXBs in Galactic globular clusters with the LMXB populations in the rest of the Galaxy or in other galaxies. Such comparisons are relevant for, e.g., constraining the effects of metallicity on the LMXB formation efficiency or the frequency of ultra-compact LMXBs (i.e. those with orbital periods $<1$ hour).

\section{Faint sources in globular and open clusters}

Globular clusters

With the Einstein Observatory and later ROSAT, X-ray sources in globular clusters could be studied with better spatial resolution and sensitivity than previously (e.g. Hertz \& Grindlay 1983, Verbunt 2001). Despite the improved resolution, positional errors were typically so large that identifying optical or UV counterparts remained challenging. Chan$d r a$ has brought about a radical change. Early Chandra observations of globular clusters revealed an overwhelming abundance and variety of faint sources; see e.g. the initial work on 47 Tuc by Grindlay et al. (2001). Four main faint $\left(L_{X} \lesssim 10^{33} \mathrm{erg} \mathrm{s}^{-1}\right)$ source classes can be distinguished in globular clusters: i) quiescent neutron-star LMXBs or qLMXBs, which are often classified as such based on their soft X-ray spectra; ii) white dwarfs accreting from low-mass companions, also called cataclysmic variables or CVs; iii) millisecond pulsars, sometimes isolated, often in binaries; iv) magnetically active binaries, which are detached or contact binaries that emit coronal $\mathrm{X}$ rays as a result of magnetic activity on a rapidly spinning main-sequence or subgiant star that is tidally locked to its companion. These active binaries or ABs are the most abundant X-ray source class in globular clusters, and are also the X-ray faintest of the four source types. The thermal $\mathrm{X}$-ray emission of qLMXBs can be valuable for constraining the equation of state of dense matter, especially when the distances are well-known as is the case for globular clusters systems (e.g. Bogdanov et al. 2016).

Until recently, it was argued that most black holes that form in a cluster are not retained for more than $\sim 1$ Gyr, but are ejected from the cluster as a result of dynamical interactions. Driven by both theoretical and observational work, this view has now changed (for a review see Kremer et al. (2019), this volume). There is no candidate stellarmass black hole among the luminous LMXBs in globular clusters (possibly because of the small overall sample). But in the past years, several candidate stellar-mass black holes have been identified both among the lower-luminosity X-ray sources (see e.g. Shishkovsky et al. 2018) and through optical surveys (the candidate found by Giesers et al. (2018) does not match with any known X-ray source). The system X 9 in 47 Tuc (Miller-Jones et al. 2015), previously classified as a bright CV, is particularly interesting, not only because of the possible black-hole nature of the accretor, but also because of its compact donor. Periodicity in the X-ray light curve of $\sim 28$ min shows it is an ultra-compact binary, which only leaves room for a degenerate donor (Bahramian et al. 2017). The optical spectrum lacks any sign of hydrogen (Tudor et al. 2018). It has been suggested that X 9 could have formed by the tidal capture of a horizontal branch star by black hole. In this scenario, the expulsion of the giant's envelope during the subsequent common-envelope phase, left a white dwarf, which has now become the mass donor (Church et al. 2017). 
Table 1. Luminous X-ray sources and transients in Galactic globular clusters ${ }^{1}$

\begin{tabular}{|c|c|c|c|c|c|}
\hline Cluster & ID & Position RA, Dec (J2000) & $P_{b}$ & Opt & Note \\
\hline Liller 1 & Rapid Burster [1] & $17^{\mathrm{h}} 33^{\mathrm{m}} 24^{\mathrm{s}} 61,-33^{\circ} 23^{\prime} 19.8^{\prime \prime}[2 ; \mathrm{R}]$ & - & - & $\mathrm{T}$ \\
\hline Liller 1 & CXOU J173324.6-332321 [3] & $17^{\mathrm{h}} 33^{\mathrm{m}} 24^{\mathrm{s}} 61,-33^{\circ} 23^{\prime} 21.2^{\prime \prime}[4 ; \mathrm{X}]$ & - & - & $\mathrm{T}$ \\
\hline Liller 1 & CXOU J173324.1-332316 [5] & $17^{\mathrm{h}} 33^{\mathrm{m}} 24^{\mathrm{s}} 14,-33^{\circ} 23^{\prime} 16.0^{\prime \prime} \quad[5 ; \mathrm{X}]$ & - & - & $\mathrm{T} ?^{a}$ \\
\hline M 15 & M $15 \mathrm{X}-1, \mathrm{AC} 211^{b}[6]$ & $21^{\mathrm{h}} 29^{\mathrm{m}} 58^{\mathrm{s}} \cdot 31,+12^{\circ} 10^{\prime} 02.89^{\prime \prime}[7 ; \mathrm{R}]$ & $17.1 \mathrm{~h}[8]$ & $+[9]$ & $\mathrm{P}$ \\
\hline M 15 & M 15 X-2 [6] & $21^{\mathrm{h}} 29^{\mathrm{m}} 58^{\mathrm{s}} \cdot 13,+12^{\circ} 10^{\prime} 02.6^{\prime \prime}[6 ; \mathrm{X}]$ & $22.6 \mathrm{~m} \mathrm{[10]}$ & $+[6]$ & $\mathrm{P}$ \\
\hline M 28 & IGR J18245-2452 [11] & $18^{\mathrm{h}} 24^{\mathrm{m}} 32^{\mathrm{s}} .51,-24^{\circ} 52^{\prime} 07.9^{\prime \prime}[12 ; \mathrm{R}]$ & $11.0 \mathrm{~h}[13]$ & $+[14]$ & $\mathrm{T}^{c}$ \\
\hline NGC 1851 & $4 \mathrm{U} 0513-40[15]$ & $05^{\mathrm{h}} 14^{\mathrm{m}} 06^{\mathrm{s}} \cdot 48,-40^{\circ} 02^{\prime} 38.8^{\prime \prime}[16 ; \mathrm{X}]$ & $\sim 17 \mathrm{~m}[17]$ & $+[18]$ & $\mathrm{P}$ \\
\hline NGC 2808 & Swift J0911-6452 [19] & $09^{\mathrm{h}} 12^{\mathrm{m}} 02^{\mathrm{s}} \cdot 46,-64^{\circ} 52^{\prime} 06 \cdot 4^{\prime \prime}[20 ; \mathrm{X}]$ & $44.3 \mathrm{~m}[21]$ & - & $\mathrm{T}$ \\
\hline NGC 6388 & IGR J17361-4441 [22] & $17^{\mathrm{h}} 36^{\mathrm{m}} 17^{\mathrm{s}} \cdot 42,-44^{\circ} 44^{\prime} 05.98^{\prime \prime} \quad[23 ; \mathrm{X}]$ & - & - & $\mathrm{T}^{d}$ \\
\hline NGC 6440 & SAX J1748.9-2021 ${ }^{e}[24]$ & $17^{\mathrm{h}} 48^{\mathrm{m}} 52^{\mathrm{s}} \cdot 2,-20^{\circ} 21^{\prime} 32.6^{\prime \prime}[25 ; \mathrm{O}]$ & $8.7 \mathrm{~h}[26]$ & $+[27,28]$ & $\mathrm{T}$ \\
\hline NGC 6440 & NGC 6440 X-2 [29] & $17^{\mathrm{h}} 48^{\mathrm{m}} 52^{\mathrm{s}} .76,-20^{\circ} 21^{\prime} 24.0^{\prime \prime} \quad[30 ; \mathrm{X}]$ & $57.3 \mathrm{~m}[31]$ & - & $\mathrm{T}$ \\
\hline NGC 6441 & $4 \mathrm{U} 1746-37[32]$ & $17^{\mathrm{h}} 50^{\mathrm{m}} 12^{\mathrm{s}} .73,-37^{\circ} 03^{\prime} 06.5^{\prime \prime} \quad[33 ; \mathrm{O}]$ & $5.2 \mathrm{~h}[34]$ & $+[35,33]$ & $\mathrm{P}$ \\
\hline NGC 6624 & $4 \mathrm{U} 1820-30[32]$ & $18^{\mathrm{h}} 23^{\mathrm{m}} 40^{\mathrm{s}} .57,-30^{\circ} 21^{\prime} 39.92^{\prime \prime} \quad[36 ; \mathrm{O}]$ & $11.4 \mathrm{~m}[37]$ & $+[36]$ & $\mathrm{P}$ \\
\hline NGC 6652 & XB $1832-330[38]$ & $18^{\mathrm{h}} 35^{\mathrm{m}} 43^{\mathrm{s}} 67,-32^{\circ} 59^{\prime} 26.3^{\prime \prime}[39 ; \mathrm{X}]$ & $2.1 \mathrm{~h}[40]$ & $+[41]$ & $\mathrm{P}^{f}$ \\
\hline NGC 6712 & $4 \mathrm{U} 1850-087[42]$ & $18^{\mathrm{h}} 53^{\mathrm{m}} 04^{\mathrm{s}} .91,-08^{\circ} 42^{\prime} 19.35^{\prime \prime}[43 ; \mathrm{O}]$ & $20.6 \mathrm{~m}[44]$ & $+[45]$ & $\mathrm{P}$ \\
\hline Terzan 1 & XB $1732-304[46]$ & $17^{\mathrm{h}} 35^{\mathrm{m}} 47^{\mathrm{s}} \cdot 26,-30^{\circ} 28^{\prime} 55.3^{\prime \prime g} \quad[47 ; \mathrm{X}]$ & - & - & $\mathrm{T}$ \\
\hline Terzan 2 & $4 \mathrm{U} 1722-30[48]$ & $17^{\mathrm{h}} 27^{\mathrm{m}} 33^{\mathrm{s}} \cdot 15,-30^{\circ} 48^{\prime} 07.8^{\prime \prime}[49 ; \mathrm{X}]$ & $--^{h}$ & - & $\mathrm{P}$ \\
\hline Terzan 5 & Terzan $5 \mathrm{X}-1^{i}[50,51]$ & $17^{\mathrm{h}} 48^{\mathrm{m}} 05^{\mathrm{s}} \cdot 23,-24^{\circ} 46^{\prime} 47.6^{\prime \prime}[52 ; \mathrm{O}]$ & - & $+[52]$ & $\mathrm{T}$ \\
\hline Terzan 5 & Terzan 5 X-2 $2^{j} \quad[53,54]$ & $17^{\mathrm{h}} 48^{\mathrm{h}} 04^{\mathrm{s}} 82,-24^{\circ} 46^{\prime} 48.9^{\prime \prime}[55 ; \mathrm{M}]$ & $21.3 \mathrm{~h}[56]$ & $+[57]$ & $\mathrm{T}$ \\
\hline Terzan 5 & Terzan $5 \mathrm{X}-3^{k}[58]$ & $17^{\mathrm{h}} 48^{\mathrm{m}} 05^{\mathrm{s}} \cdot 41,-24^{\circ} 46^{\prime} 38.0^{\prime \prime} \quad[59 ; \mathrm{X}]$ & - & - & $\mathrm{T}$ \\
\hline Terzan 6 & GRS $1747-312$ [60] & $17^{\mathrm{h}} 50^{\mathrm{m}} 46^{\mathrm{s}} .86,-31^{\circ} 16^{\prime} 28.9^{\prime \prime}[61 ; \mathrm{X}]$ & $12.4 \mathrm{~h}[61]$ & - & $\mathrm{T}$ \\
\hline
\end{tabular}

${ }^{1}$ Status as of August 2019. The column "ID" gives (one of) the common source name(s) and a reference to an early report of the detection as a persistent or transient luminous source. The column "Position" gives a recent accurate position reported in the literature and the method to measure it: $\mathrm{M}=$ Moon occultation, $\mathrm{O}=\mathrm{optical}$, $\mathrm{R}=$ Radio, $\mathrm{X}=\mathrm{X}$-ray. The column " $P_{b}$ " gives the orbital period, and the entry in the column "Opt" indicates whether an optical (or near-ultraviolet, or near-infrared) counterpart has been identified $(+)$ or not $(-)$. The column "Note" has additional comments and indicates whether a source has been persistently luminous since its discovery $(\mathrm{P})$, or whether it is transient $(\mathrm{T}){ }^{a}$ The Chandra position of the faint $\left(L_{X}(0.5-2.5 \mathrm{keV}) \approx 10^{32-34}\right.$ $\mathrm{erg} \mathrm{s}^{-1}$ ) source $\mathrm{C} 1$ in [5] is a positional match to the Einstein position reported for the X-ray source in Liller 1 (Grindlay et al. 1984), suggesting that $\mathrm{C} 1$ could be a third transient the cluster. ${ }^{b} \mathrm{AC} 211$ is the name of the optical counterpart to $4 \mathrm{U} 2127+119$ [9], which is the bright X-ray source in M 15 that was later resolved into M 15 X-1 and M 15 X-2 by [6]. ${ }^{c}$ transitional MSP, switches between a rotation-powered radio pulsar state to an accretion-powered X-ray pulsar state [13] ${ }^{d}$ Del Santo et al. (2014) suggest the source of the transient X-ray emission could be the tidal disruption of a planet by a white dwarf, but Wijnands et al. (2015) argue it is more likely that this source is an unusual LMXB. ${ }^{e} \mathrm{X}$-ray emission from NGC 6440 was already detected in the 1970 s, but it is unclear which X-ray source was responsible for this emission. ${ }^{f}$ Johnston et al. (1996) refer to the source as a "bright X-ray transient". ${ }^{g}$ This position is for the Chandra source CX 2, the most likely quiescent counterpart to the luminous source that has dimmed significantly since the second half of the $1990 \mathrm{~s} .{ }^{h}$ candidate ultra-compact binary (in 't Zand et al. 2007) ${ }^{i}$ An often-used name for this transient is EXO 1745-248, which is a little unfortunate since it is unclear whether Terzan 5 X-1 is the same source that was detected by EXOSAT. ${ }^{j}$ also known as IGR J17480-2446 ${ }^{k}$ also known as Swift J174805.3-244637.

References: [1] Lewin et al. 1976, IAUC 2922; [2] Moore et al. 2000, ApJ, 532, 1181; [3] Homan et al. 2018, ATel 11598; [4] Bahramian et al. 2018, ATel 11646; the Chandra position from [3] was adjusted so that the position for the Rapid Burster from [3] matches the more accurate radio position from [2]; [5] Homer et al. 2001, AJ, 122, 2627; [6] White \& Angelini 2001, ApJ, 561, L101; [7] Kulkarni et al. 1990, ApJ, 363, L5; [8] Ilovaisky et al. 1993, A\&A, 270, 139; [9] Aurière et al. 1984, A\&A, 138, 415; [10] Dieball et al. 2005, ApJ, 634, L105; [11] Eckert et al. 2013, ATel 4925; [12] Pavan et al. 2013, ATel 4981; [13] Papitto et al. 2013, Nature, 501, 517; [14] Pallanca et al. 2013, ATel 5003; [15] Clark et al. 1975, ApJ, 199, L93; [16] Fiocchi et al. 2011, MNRAS, 414, L41; [17] Zurek et al. 2009, ApJ, 699, 1113; [18] Homer et al. 2001, ApJ, 550, L155; [19] Serino et al. 2016, ATel 8862; [20] Homan et al. 2016, ATel 8971; [21] Sanna et al. 2017, A\&A, 598, A34; [22] Gibaud et al. 2011, ATel 3565; [23] Pooley et al. 2011, ATel 3627; [24] in 't Zand et al. 1999, A\&A, 345, 100; [25] Cadelano et al. 2017, ApJ, 844, 53; [26] Altamirano et al. 2008, ApJ, 674, L45; [27] Verbunt et al. 2000, A\&A, 359, 960; [28] in 't Zand et al. 2001, ApJ, 563, L41; [29] Heinke \& Budac 2009, ATel 2139; [30] Heinke et al. 2010, ApJ, 714, 894; [31] Altamirano et al. 2010, ApJ, 712, 58; [32] Giacconi et al. 1974, ApJS 27, 37; [33] Homer et al. 2002, AJ, 123, 3255; [34] Balucińska-Church et al. 2004, MNRAS, 334, 338; [35] Deutsch et al. 1998, ApJ, 493, 775, NGC6441; [36] King et al. 1993, ApJ, 413, L117; [37] Stella et al. 1987, ApJ, 312, L17; [38] Hertz \& Wood 1985, ApJ, 290, 171; [39] Stacey et al. 2012, ApJ, 751, 62; [40] Engel et al. 2012, ApJ, 747, 119; [41] Heinke et al. 2001, ApJ, 562, 363; [42] Seward et al. 1976, MNRAS, 175, 39P; [43] Ferraro et al. 2000, ApJ, 542, L29; [44] Homer et al. 1996, MNRAS, 282, L37; [45] Bailyn et al. 1991, ASP Conf. Series, 13, 363; [46] Makishima et al. 1981, ApJ, 247, L23; [47] Cackett et al. 2006, MNRAS, 369, 407; [48] Swank et al. 1977, ApJ 212, L73; [49] Revnivtsev et al. 2002, Astronomy Letters, 28, 237; [50] Markwardt \& Swank 2010, IAUC 7454; [51] Heinke et al. 2003, ApJ, 590, 809; [52] Ferraro et al. 2015, ApJ, 807, L1; [53] Bordas et al. 2010, ATel 2919; [54] Pooley et al. 2010, ATel 2974; [55] Riggio et al. 2012, ApJ, 754, L11; [56] Papitto et al. 2011, A\&A, 526, L3; [57] Testa et al. 2012, A\&A, 547, A28; [58] Wijnands et al. 2012, ATel 4242; [59] Bahramian et al. 2014, ApJ, 780, 127; [60] Predehl et al. 1991, A\&A, 246, L21; [61] in 't Zand et al. 2003, A\&A, $406,233$. 
Old open clusters

Before Chandra, several nearby $(\lesssim 1 \mathrm{kpc})$ old $(\lesssim 4$ Gyr $)$ open clusters had already been studied with ROSAT (e.g. Belloni et al. 1998, Belloni \& Tagliaferri 1998), but Chandra and also XMM-Newton have brought the more distant - and often older-open clusters within reach (e.g. Gosnell et al. 2012, van den Berg et al. 2013, Vats \& van den Berg 2017, Vats et al. 2018). Not only does this provide an opportunity to study more comprehensively if, and how, the X-ray-source population in open clusters evolves in time; it also narrows the age gap that exists in X-ray studies of old open and globular clusters, and offers a view how e.g. cluster structure, stellar density and metallicity may affect an old cluster's X-ray emission. Most X-ray sources in old open clusters are ABs, and some CVs have been detected as well. It is not surprising that no confirmed LMXBs (neither luminous nor quiescent) have been found in old open clusters, where present-day stellar densities are too low to create them dynamically. Although the number density of LMXBs in the Galactic field is uncertain, the total mass represented by the Galactic old open clusters is very likely too low to expect any primordial LMXBs. A group of cluster X-ray sources that are poorly understood are the sub-subgiants, and the blue and yellow stragglers. These are found in regions of the color-magnitude diagram that are not occupied by single or binary cluster stars that have followed "standard" evolution paths. Their X-ray properties resemble those of $\mathrm{ABs}$, and while their formation and present evolutionary state may be unclear, the X-rays may be the result of rotation-enhanced magnetic activity - e.g. due to tidal interaction, or perhaps spin-up after an episode of accretion or a merger event. The latter scenario was suggested to explain the X-rays from the yellow straggler S 1237 in M 67, which is likely composed of an evolved blue straggler and a turn-off star (or another blue straggler) in a 698 day orbit (Leiner et al. 2016). It is interesting to point out that X-ray sources like S 1237 have not been found in globular clusters (yet). This may be the result of the higher stellar densities in globular clusters, which could cause binaries in orbits as wide as that of S 1237 to be disrupted.

\section{Source classification}

Our current understanding of the faint X-ray sources (or, more generally, of the close binaries) in old clusters can partly be attributed to detailed complementary work at other wavelengths. For example, optical and/or (near-)ultraviolet (NUV) work with HST has been indispensable to identify, classify and characterize Chandra sources (e.g. Edmonds et al. 2003a, Edmonds et al. 2003b, Cadelano et al. 2017, Hare et al. 2018, Zhao et al. 2019). Even with Chandra, the source error circles in globular clusters often contain more than one candidate counterpart. Looking for blue, $H \alpha$-bright or variable $H S T$ counterparts inside the small Chandra error circles has proven to be a powerful way to identify the true counterparts. Accreting binaries usually stand out in their optical/NUV colors that are typically much bluer, or brighter in $H \alpha$, due to the contribution from an accretion disk, or (in CVs) from the white dwarf itself. ABs as well show $H \alpha$-excess emission from an active chromosphere. For open clusters, the WOCS survey (Mathieu 2000) has given valuable information on photometry, radial velocities, and proper motions for a select number of clusters. With the help of Gaia proper motions, membership can now be established for X-ray sources in a much larger number of open clusters, provided the optical counterparts can be confidently identified.

Also without a multi-wavelength approach we can get some insight into the nature of the X-ray sources, even if they are too faint to constrain their X-ray spectral properties. At a distance of $\sim 1.8 \mathrm{kpc}, \mathrm{M} 4$ is the globular cluster nearest to us. The Chandra observations of M 4 are the deepest X-ray images of a globular cluster so far. With a combined sensitivity of several times $10^{28} \mathrm{erg} \mathrm{s}^{-1}$, these observations are especially suited to study 
ABs. Only the observations of 47 Tuc and NGC 6397 come close to this limit (but they do not reach it); X-ray studies of other clusters do not go deeper than, typically, $10^{30-31} \mathrm{erg}$ $\mathrm{s}^{-1}$. Pooley (2016) gives a preview of the M 4 results, showing (in their Fig. 2) that the radial distribution of $\mathrm{X}$-ray sources within the half-mass radius is shallower than those in 47 Tuc and NGC 6397. Further analysis shows that this can largely be attributed to sources fainter than $\sim 10^{29} \mathrm{erg} \mathrm{s}^{-1}$, which lie below the 47 Tuc and NGC 6397 detection limit. This implies that those faint sources are overall less massive, if the cluster relaxation time scale is short enough for mass segregation to take effect. This is as expected if those fainter sources are dominated by ABs, as opposed to CVs, MSPs or qLMXBs, which are typically heavier because they contain a compact object.

\section{Effects of dynamical encounters}

With a larger sample of faint sources, it became possible to investigate whether the correlation between source numbers and encounter rate also extends to the qLMXBs and other faint source classes in globular clusters. A correlation was indeed found for the qLMXBs (e.g. Heinke et al. 2003, Pooley et al. 2003). Pooley \& Hut (2006) were the first to show observationally that dynamics likely also plays a role in the formation of CVs. On the other hand, various authors (e.g. Bassa et al. 2008, Lu et al. 2009) used Chandra observations to show that globular clusters contain primordial binaries as well. The level of dynamical activity is expected to be much lower in old open clusters, but the effect of encounters cannot be ignored altogether with compelling evidence coming from blue stragglers. Detailed, long-term observations indicate that the majority of blue stragglers in NGC 188, M 67, and NGC 6819 formed as a result of mass transfer in close binaries (see Geller (2016) for a summary and references). However, there are also several blue stragglers (including some faint X-ray sources) for which encounters probably played a role in their formation (e.g. van den Berg et al. 2001, Mathieu \& Geller 2009).

More detailed insights into how dynamics may affect CVs in globular custers has come from a comparison of Chandra-selected CVs in the core-collapsed clusters NGC 6397 and NGC 6752 (Cohn et al. 2010, Lugger et al. 2017), with those in the non-collapsed cluster 47 Tuc (Rivera Sandoval et al. 2018). The CVs in 47 Tuc show a single-peaked distribution of their absolute optical magnitudes, whereas the CVs in the core-collapsed clusters show bimodal distributions. In the latter, the bright CVs are also found to be more massive and more concentrated towards the cluster center than the faint ones. It was suggested that the bright CVs are still young, and were dynamically formed near the center of the core-collapsed clusters where interactions are assumed to occur frequently. The fainter systems, on the other hand, are old (and therefore less luminous as the donor masses dwindle and the accretion rates drop), and less concentrated as a result of one or more scattering encounters that pushed them further out. But recent numerical simulations have provided a different perspective. Belloni et al. (2019) find that encounters only play a limited role in the formation of CVs, and that most CVs that are currently observed, evolved from primordial binaries. According to their results, the bimodal magnitude distribution is mainly the natural result of $\mathrm{CV}$ evolution driven by angular momentum loss, where the young systems are still more bright and massive and have longer periods, and the older systems have already evolved to short periods and have become fainter and less massive. Whether these bright CVs show a different radial distribution than the faint ones is determined by the relaxation time of the host globular clusters. If it is short (as is the case in core-collapsed clusters), the bright systems will naturally show a different, more concentrated distribution than the faint ones, since mass segregation has had time to drive them to different locations in the cluster. The fact that the contribution of primordial CVs to the total observable CV population exceeds the contribution from 
dynamically formed CVs, then implies that the correlation between the number of CVs and the normalized (to the cluster mass) encounter rate should be weak or absent. This appears to be at odds with the findings by Pooley \& Hut (2006). Another outcome of these simulations is that the destruction of $\mathrm{CV}$ progenitors, which are wide main-sequence binaries, outweighs the dynamical production of new CVs.

Do we see observational proof for binary destruction or disruption? A number of results may indeed point in that direction. By parameterizing the probability that a binary experiences an encounter after it has been dynamically formed $(\gamma)$, Verbunt \& Freire (2014) showed that $\gamma$ reasonably describes which clusters are more likely to host radio pulsars that appear to have been affected by secondary encounters (e.g isolated or slow pulsars, or products of exchange encounters). The analysis of globular-cluster Fermi data by De Menezes et al. (2019) suggests a similar correlation, in the sense that the inferred number of MSPs goes down for clusters with higher $\gamma$ values (suggesting that potential progenitor systems are disrupted before an MSP is formed). We can also consider the issue of binary destruction in terms of the overall number of X-ray sources per unit mass. As has been shown by e.g. Bassa et al. (2008), the number of X-ray sources scales with both the cluster mass (reflecting the primordial binaries) and the rate of encounters (reflecting dynamically formed sytems). Fig. 9 in Lan et al. (2010) is a graphical representation of this result. Going down to the regime of small cluster mass and few encounters where clusters like E 3 or NGC 6535 are found, no or very few X-ray sources are observed and expected (Lan et al. 2010, Kong et al. private communication). The present-day stellar densities in old open clusters are much lower still. Extrapolating from the globular clusters, and applying the same X-ray luminosity limit, we do not expect to see any Xray sources in old open clusters. But in fact, many ABs and some CVs that are solid cluster members have been identified in old open clusters. Therefore, old open clusters have more X-ray sources than the lowest-density globular clusters, which are similar in mass or only several times more massive (and, as outlined in Section 4 this difference also persists for higher-density globular clusters). Since the current rate of encounters in these sparse globular clusters is very low, it is not clear whether binary destruction is the (only) cause for this discrepancy. On the other hand, it is important to keep in mind that the encounter rate that is estimated from the current cluster parameters may be very different from the rate in the past, when the cluster core may have been much denser.

\section{X-ray emissivity}

We can look at the X-ray emission of old star clusters in a larger context and, instead of studying sources individually, compare the combined X-ray emission from old stellar populations in general. The combined X-ray emission per unit of mass, or X-ray emissivity, has been measured for other environments besides globular and old open clusters, such as various other parts of the Galaxy (the local neighborhood, parts of the bulge, and the Galactic center region-e.g. Sazonov et al. 2006, Muno et al. 2006, Revnivtsev et al. 2007a, Hong et al. 2009), and extragalactic populations (e.g. Revnivtsev et al. 2007b, Ge et al. 2015). However, due to the different analysis methods and adopted energy bands, it is often not straightforward to compare the results of these studies.

Using ROSAT observations, Verbunt (2001) showed for a large sample of globular clusters that they are less X-ray luminous per unit mass than the open cluster M 67 (when luminous X-ray binaries are disregarded), possibly/partly due to binary destruction, differences in metallicity or age. Also, as a result of the shorter relaxation times of open clusters, and of their orbits in or through the Galactic plane, open clusters lose stars 
at a faster rate than globular clusters. This could artificially boost the X-ray emission per unit of mass that we see today if the binaries that evolve to become X-ray sources are preferentially kept. Of all old open clusters studied at the time, the X-ray source population of M 67 was best characterized. Based on deeper Chandra observations, and taking advantage of better source classifications, van den Berg et al. (2013) looked at this in more detail and compared the number of CVs and $\mathrm{ABs}$ (with $L_{X} \gtrsim 10^{30}$ erg $\mathrm{s}^{-1}$ and inside the half-mass radius) per unit of mass in the old open clusters M67, NGC 6819, and NGC 6791 and the globular clusters 47 Tuc and NGC 6397. They found that both CVs and ABs are underabundant in globular clusters. On the other hand, they also showed that M67 may be an outlier among the old open clusters, having relatively more ABs than the other open clusters. This underlined the need to study the X-ray source populations in more old open clusters to be able to put any conclusions on firmer grounds.

Ge et al. (2015) investigated the X-ray emissivities of a somewhat larger, but still quite limited, number of old open and globular clusters but extended the comparison to environments where dynamical interactions are presumably irrelevant. Excluding old extragalactic populations where hot gas or recent star formation contribute to the X-ray emission, they find that the X-ray emissivities of dwarf galaxies, the bulge of M 31 and the local neighborhood are consistent. From this they conclude there may be a quasiuniversal value of the X-ray emissivity for old, dynamically inactive stellar populations. Although their results show that the X-ray emission of most globular clusters in their small sample (47 Tuc, NGC 6397, NGC 6266) also agree with this value, they acknowledge that more globular clusters need to be studied in order to understand whether and how cluster dynamics could boost or suppress the X-ray emission. The open clusters M 67 and NGC 6791 were again found to be more X-ray luminous than the other environments with both higher and lower stellar densities. This implies that the observed differences in Xray emissivity cannot be attributed to the dynamical destruction of binaries (alone). The other outlier in the Ge et al. sample is $\omega$ Cen; however, this cluster may have a very different origin than the other Galactic globular clusters.

Cheng et al. (2018) compare the X-ray emissivity of a large sample of Galactic globular clusters. Specifically, their work looks at the correlation with encounter rate (per unit of mass), and revisits the earlier result by e.g. Pooley \& Hut (2006) that more X-ray sources are found in clusters where one expects more interactions. According to the analysis by Cheng et al., the trend of increasing X-ray emissivity with encounter rate becomes much less significant, which they take as a sign that the contribution of primordial binaries is more important than previously thought. This finding is in line with the theoretical findings by Belloni et al. (2019) whose numerical simulations indicate that the primordial CVs dominate the present-day sample of observable CVs (see last part of Section 3). A new study by Heinke et al. (in preparation) also analyzes the X-ray emissivity of a large sample of globular clusters and several old open clusters, focusing on investigating possible correlations with metallicity, age and binary fraction.

In summary, studies of the collective X-ray emission of old star clusters and detailed investigations of individual sources complement each other to reach a better understanding of the internal cluster dynamics, binary evolution, and the overall population of short-period (i.e. X-ray-emitting) binaries.

\section{References}

Arnason, R., Sivakoff, G., Heinke, C., Cohn, H., Lugger, P. 2015, ApJ, 807, 52 
Bagchi, M., Lorimer, D., Chennamangalam, J. 2011, MNRAS, 418, 477

Bahramian, A., Heinke, C., Sivakoff, G., Gladstone, J. 2013, ApJ, 766, 136

Bahramian, A., Heinke, C., Sivakoff, G., Altamirano, D., Wijnands, R., Homan, J., Linares, M., Pooley, D., Degenaar, N., Gladstone, J. 2014, ApJ, 780, 127

Bahramian, A., Heinke, C., Tudor, V., Miller-Jones, J., Bogdanov, S., Maccarone, T., Knigge, C., Sivakoff, G., Chomiuk, L., Strader, J., Garcia, J., Kallman, T. 2017, MNRAS, 467, 2199

Bassa, C., Pooley, D., Verbunt, F., Homer, L., Anderson, S., Lewin, W. 2008, A\& A, 488, 921

Belloni, D., Giersz, M., Rivera Sandoval, L., Askar, A., Ciecielåg, P. 2019, MNRAS, 483, 315

Belloni, T. \& Tagliaferri, G. 1998, A\&A, 335, 517

Belloni, T., Verbunt, F., Mathieu, R. 1998, A\&A, 339, 431

Blumenthal, G. \& Tucker, W. 1974, ARAA, 12, 23

Bogdanov, S., Heinke, C., Özel, F., Güver, T. 2016, ApJ, 831, 184

Cadelano, M., Pallanca, C., Ferraro, F., Dalessandro, E., Lanzoni, B., Patruno, A. 2017, ApJ, 844,53

Carbone, D. \& Wijnands, R. 2019, MNRAS, 488, 2767

Cheng, Z., Li, Z., Xu, X., Li, X. 2018, ApJ, 858, 33

Church, R., Strader, J., Davies, M., Bobrick, A. 2017, ApJ, 851, L4

Clark, G., Markert, T., Li, F. 1975, ApJ, 199, L93

Clark, G., Jernigan, J., Bradt, H., Canizares, C., Lewin, W., Li, F., Mayer, W., McClintock, J., Schnopper, H. 1976, ApJ, 207, L105

Cohn, H., Lugger, P., Couch, S., Anderson, J., Cool, A., van den Berg, M., Bogdanov, S., Heinke, C., Grindlay, J. 2010, ApJ, 722, 20

Del Santo, M., Nucita, A., Lodato, G., Manni, L. De Paolis, F., Farihi, J., De Cesare, G., Segreto, A. 2014, MNRAS, 444, 93

de Menezes, R., Cafardo, F., Nemmen, R. 2019, MNRAS, 486, 851

Edmonds, P., Gilliland, R., Heinke, C., Grindlay, J. 2003a, ApJ, 596, 1177

Edmonds, P., Gilliland, R., Heinke, C., Grindlay, J. 2003b, ApJ, 596, 1197

Ge, C., Li, Z., Xu, X., Gu, Q., Wang, Q., Roberts, S., Kraft, R., Jones, C., Forman, W. 2015, ApJ, 812, 130

Geller, A. 2016, Mem. S. A. It., 87, 505

Giacconi, R., Murray, S., Gursky, H., Kellogg, E., Schreier, E., Matilsky, T., Koch, D., Tananbaum, H., 1974, ApJS, 27, 37

Giesers, B., Dreizler, S., Husser, T., Kamann, S., Anglada Escudé, G., Brinchmann, J., Carollo, C., Roth, M., Weilbacher, P., Wisotzki, L. 2018, MNRAS, 475, L15

Gosnell, N., Pooley, D., Geller, A., Kalirai, J., Mathieu, R., Frinchaboy, P., Ramirez-Ruiz, E. 2012, ApJ, 745, 57

Grindlay, J. \& Heise, J. 1975, IAUC, 2879

Grindlay, J., Hertz, P., Steiner, J., Murray, S., Lightman, A. 1984, ApJ, 282, L13

Grindlay, J., Heinke, C., Edmonds, P., Murray, S. 2001, Science, 292, 2290

Hare, J., Kargaltsev, O., Rangelov, B. 2018, ApJ, 865, 33

Heinke, C., Grindlay, J., Lugger, P., Cohn, H., Edmonds, P., Lloyd, D., Cool, A. 2003, ApJ, 598,501

Heinke, C., Cohn, H., Lugger, P. 2009, ApJ, 692, 584

Heinke, C. 2010, American Institute of Physics Conference Series, 1314, 135

Hertz, P. \& Grindlay, J. 1983, ApJ, 275, 105

Homan, J., van den Berg, M., Heinke, C., Pooley, D., Degenaar, N., van den Eijnden, J., Bahramian, A., Gendreau, K., Arzoumanian, Z. 2019, ATel, 11598

Hong, J., van den Berg, M., Grindlay, J., Laycock, S. 2009, ApJ, 706, 223

in 't Zand, J., Jonker, P., Markwardt, C. 2007, A\&A, 465, 953

Ivanova, N. 2017, in: Star clusters and black holes in galaxies across cosmic time, IAU Symposium 312, arXiv:1706.07578

Johnston, J., Verbunt, F., Hasinger, G. 1996, A\&A, 309, 116

Katz, J. 1975, Nature, 253, 698

Kremer, K., Ye, C., Chatterjee, S., Rodriguez, C., Rasio, F. 2019, to appear in this volume, arXiv:1907.12564 
Lan, S., Kong, A., Verbunt, F., Lewin, W., Bassa, C., Anderson, S., Pooley, D. 2010, ApJ, 712, 380

Leiner, E., Mathieu, R., Stello, D., Vanderburg, A., Sandquist, E. 2016, ApJ, 2016, 832, L13

Lewin, W., van Paradijs, J., Taam, R 1995, in: X-ray Binaries, Cambridge Astrophysics Series, Cambridge, MA: Cambridge University Press, edited by Lewin, W., van Paradijs, J. \& van den Heuvel, E.

Lu, T.-N., Kong, A., Bassa, C., Verbunt, F., Lewin, W., Anderson, S., Pooley, D. 2009, ApJ, 705,175

Lugger, P., Cohn, H., Cool, A., Heinke, C., Anderson, J. 2017, ApJ, 841, 53

Mathieu, R. 2000, in: Stellar Clusters and Associations: Convection, Rotation, and Dynamos, ASP Conference Series, edited by Pallavicini, R.,Micela, G., Sciortino, S.

Mathieu, R. \& Geller, A. 2009, Nature, 462, 1032

Miller-Jones, J., Strader, J., Heinke, C., Maccarone, T., van den Berg, M., Knigge, C., Chomiuk, L., Noyola, E., Russell, T., Seth, A., Sivakoff, G. 2015, MNRAS, 453, 3918

Muno, M., Bauer, F., Bandyopadhyay, R., Wang, Q. 2006, ApJS, 165, 173

Papitto, A., Ferrigno, C., Bozzo, E., Rea, N., Pavan, L., Burderi, L., Burgay, M., Campana, S., di Salvo, T., Falanga, M., Filipović, M., Freire, P., Hessels, J., Possenti, A., Ransom, S., Riggio, A., Romano, P., Sarkissian, J., Stairs, I., Stella, L., Torres, D., Wieringa, M., Wong, G. 2013, Nature, 501, 517

Pooley, D., Lewin, W., Anderson, S., Baumgardt, H., Filippenko, A., Gaensler, B., Homer, L., Hut, P., Kaspi, V., Makino, J., Margon, B., McMillan, S., Portegies Zwart, S., van der Klis, M., Verbunt, F. 2003, ApJ, 591, L131

Pooley, D. \& Hut, P. 2006, ApJ, 646, L143

Pooley, D. 2016, Mem.S. A. It., 87, 547

Revnivtsev, M., Vikhlinin, A., Sazonov, S. 2007a, A\&A, 473, 857

Revnivtsev, M., Churazov, E., Sazonov, S., Forman, W., Jones, C. 2007b, A\&A A, 473, 783

Rivera Sandoval, L., van den Berg, M., Heinke, C., Cohn, H., Lugger, P., Anderson, J., Cool, A., Edmonds, P., Wijnands, R., Ivanova, N., Grindlay, J. 2018, MNRAS, 475, 4841

Sazonov, S., Revnivtsev, M., Gilfanov, M., Churazov, E., Sunyaev, R. 2006, A 6 A, 450, 117

Shishkovsky, L., Strader, J., Chomiuk, L., Bahramian, A., Tremou, E., Li, K., Salinas, R., Tudor, V., Miller-Jones, J., Maccarone, T., Heinke, C., Sivakoff, G. 2018, ApJ, 855, 55

Tudor, V., Miller-Jones, J., Knigge, C., Maccarone, T., Tauris, T., Bahramian, A., Chomiuk, L., Heinke, C., Sivakoff, G., Strader, J., Plotkin, R., Soria, R., Albrow, M., Anderson, G., van den Berg, M., Bernardini, F., Bogdanov, S., Britt, C., Russell, D., Zurek, D. 2018, MNRAS, 476, 1889

van den Berg, M., Orosz, J., Verbunt, F., Stassun, K. 2001, A\&A, 375, 375

van den Berg, M. 2013, in: 370 Years of Astronomy in Utrecht, edited by Pugliese, G., de Koter, A., Wijburg, M., ASPC Series, 470, 251

van den Berg, M., Verbunt, F., Tagliaferri, G., Belloni, T., Bedin, L., Platais, I. 2013, ApJ, 770, 98

Vats, S. \& van den Berg, M. 2017, ApJ, 837, 130

Vats, S., van den Berg, M., Wijnands, R. 2018, MNRAS, 481, 3708

Verbunt, F. \& Hut, P. 1987, in: The Origin and Evolution of Neutron Stars, edited by Helfand, D. \& Huang, J., IAU Symposium 125, 187

Verbunt, F. 2001, A\& $A, 368,137$

Verbunt, F. and Lewin, W. 2006, in: Compact stellar X-ray sources, Cambridge Astrophysics Series, Cambridge, UK: Cambridge University Press, edited by Lewin W. \& van der Klis, M.

Verbunt, F. \& Freire, P. 2014, A\&A, 561, A11

White, N. \& Angelini, L. 2001, ApJ, 561, L101

Wijnands, R., in't Zand, J., Rupen, M., Maccarone, T., Homan, J., Cornelisse, R., Fender, R., Grindlay, J., van der Klis, M., Kuulkers, E., Markwardt, C., Miller-Jones, J., Wang, Q. 2006, A\&A A 449, 1117

Wijnands, R., Degenaar, N., Armas Padilla, M., Altamirano, D., Cavecchi, Y., Linares, M., Bahramian, A., Heinke, C. 2015, MNRAS, 454, 1371

Zhao, Y., Heinke, C., Cohn, H., Lugger, P., Cool, A. 2019, MNRAS, 483, 4560 